\title{
Autorreflexão e insight como dimensões da autoconsciência privada: uma revisão da literatura
}

\author{
Cristyan Karla Nogueira Leal ${ }^{1}$ \\ Maria Dolores Pinheiro de Souza ${ }^{1}$ \\ Mariane Lima de Souza ${ }^{1}$ \\ ${ }^{1}$ Universidade Federal do Espirito Santo, ES, Brasil
}

\begin{abstract}
Resumo
Investigações da autoconsciência têm envolvido o uso de escalas na aferição de suas dimensões e relação com cognição e comportamento, como a Escala de Autorreflexão e Insight (EAI), desenvolvida para mensurar a dimensão privada da autoconsciência. $\mathrm{O}$ instrumento parte do pressuposto de que autorreflexão e insight, componentes metacognitivos, estariam envolvidos no processo de refletir, avaliar e regular os próprios pensamentos, sentimentos e comportamentos. Esta revisão sistemática procurou entender a relação entre autorreflexão e insight na cognição e no comportamento por meio da análise de estudos empíricos que utilizaram a EAI, publicados entre 2002 e 2017, disponíveis no Portal de Periódicos CAPES, e recuperados a partir dos termos "autoconsciência"; "autorreflexão"; "EAI", e seus equivalentes em inglês, incluindo também "insight". Resultados apontam para uma associação entre insight e reações saudáveis, porém divergem sobre os benefícios da autorreflexão. Essa relação e os aspectos que a influenciam são discutidos.
\end{abstract}

Palavras-chave: Autoconsciência privada; Autorreflexão; Insight; Escala de Autorreflexão e Insight

\section{Self-reflection and insight as dimensions of private self-consciousness: a literature review}

\begin{abstract}
Research about self has involved the use of scales in measuring their dimensions and relationships with cognition and behaviour. Among those is the Self-Reflection and Insight Scale (SRIS), developed to measure the private dimension of self-consciousness. As metacognitive components, self-reflection and insight would be involved in the process of reflecting on one's own thoughts, feelings and behaviours, evaluating and modifying them in order to self-regulate. This systematic review sought to understand the relationship between self-reflection and insight into cognition and behaviour through the analysis of empirical studies that used SRIS, published between 2002 and 2017, and retrieved from Periódicos CAPES Portal, by using the words "self-consciousness", "self-awareness", "self-reflection", "SRIS", their equivalents in Portuguese, and "insight". Results pointed out an association between insight and healthy reactions but diverged on the self-reflection benefits. This relationship and the aspects that influence it are discussed.
\end{abstract}

Keywords: Private self-consciousness; Self-reflection; Insight; Self-Reflection and Insight Scale

\section{Autoreflexión e insight como dimensiones de la autoconciencia privada: uma revisión de la literatura}

\section{Resumen}

Investigaciones de la autoconciencia han usado escalas en la medición de sus dimensiones y su relación con la cognición y el comportamiento. Entre ellas se encuentra la Escala de Autoreflexión e Insight (EAI), desarrollada para medir la dimensión privada de la autoconciencia. Elementos metacognitivos, autoreflexión e insight estarían implicados en el proceso de reflexión sobre los propios pensamientos, sentimientos y comportamientos, evaluarlos y regularlos. Esta revisión sistemática trató de comprender la relación entre autoreflexión e insight en la cognición y el comportamiento a través del análisis de estudios empíricos que utilizaron la EAI, publicados entre 2002 y 2017, disponibles en el Portal de Periódicos CAPES, y recuperados a partir de las palabras "autoconsciência"; "autorreflexão", "EAI", sus equivalentes en inglés, y también "insight". Resultados apuntan a una asociación entre insight y reacciones saludables, pero difieren en los beneficios de la autoreflexión. Se discute esta relación y sus aspectos.

Palabras clave: Autoconciencia privada; Autoreflexión; Escala de Autoreflexión e Insight. 


\section{Introdução}

O fenômeno da autoconsciência é um dos temas que há séculos incita pensadores e cientistas de diversas áreas de conhecimento. Apesar da falta de consenso em torno de explicação única e de nomeação precisa, os pesquisadores parecem concordar com o fato de se tratar de um construto complexo e multidimensional. Enquanto variável psicológica, a autoconsciência pode ser compreendida como um processo humano típico e intencional que conjuga eventos internos e externos e permite a expressão de pensamentos e sentimentos em experiências ou atos individuais e coletivos por meio do corpo (DeSouza \& Gomes, 2005), tornando o indivíduo produtor e também produto de realidades e interações sociais. Sob a perspectiva da psicologia social e da personalidade, os estudiosos têm se dedicado, principalmente, ao desenvolvimento de medidas de autorrelato com o intuito de identificar e quantificar dimensões e características individuais da autoconsciência que podem influenciar a cognição e o comportamento humano.

Fenigstein, Scheier e Buss (1975) foram os pioneiros na proposição de um instrumento com essa finalidade, a Escala de Autoconsciência (EAC), inaugurando discussões subsequentes sobre os aspectos que compunham o fenômeno da autoconsciência (Anderson, Bohon, \& Berrigan,1996; Burnkrant \& Page, 1984; Creed \& Funder, 1998; Grant, Franklin, \& Langford, 2002; Trapnell \& Campbell, 1999; Watson, Morris, Ramsey, Hickman, \& Waddell, 1996) e a possibilidade real de mensurá-los (Silvia, 1999). Baseados na teoria do foco atencional de Shelley Duval e Robert Wicklund, de 1972, conhecida como Teoria da Autoconsciência ou "Objective Self-awareness Theory" (Silvia \& Duval, 2001), Fenigstein et al. defendem que, a depender da personalidade, o indivíduo tende a dirigir a atenção para aspectos internos (privados) ou externos de si mesmo (públicos), em maior ou menor grau, e afetar-se pelas consequências dessa escolha.

Para esses autores, a EAC seria capaz de mensurar três fatores ou dimensões da autoconsciência, a saber: autoconsciência privada, autoconsciência pública e ansiedade social. A primeira dimensão caracteriza indivíduos com perfil introspectivo, que tendem a orientar sua atenção aos próprios pensamentos, comportamentos e emoções. A segunda privilegia o interesse e a preocupação que se tem de si enquanto um objeto ou estímulo social, e está mais presente naqueles que agem influenciados pela opinião alheia. A terceira, relacionada à dimensão anterior, seria uma reação a esse processo de voltar o foco para si e o desconforto sentido diante de outros (Fenigstein, Scheier, \& Buss, 1975).
Incontestáveis foram as contribuições e a popularização da EAC em diversos países (DaSilveira, DeCastro, \& Gomes, 2012; Zanon \& Teixeira, 2006). Apesar disso, o trabalho de Fenigstein et al. (1975) foi alvo de críticas (Anderson et al. 1996; Burnkrant \& Page, 1984; Watson et al., 1996), revisões (Scheier \& Carver, 1985) e aprimoramentos, principalmente em torno da possível bifatorialidade da dimensão privada da autoconsciência (Anderson et al., 1996; Burnkrant \& Page, 1984; Creed \& Funder, 1998; Grant et al., 2002; Trapnell \& Campbell, 1999). Tal bifatorialidade estaria na raiz da associação da autoconsciência privada com aspectos ora positivos, ora negativos do funcionamento psíquico (Joireman, Parrot, \& Hammersla, 2002; Trapnell \& Campbell, 1999; Zanon \& Teixeira, 2006).

De fato, duas dimensões do aspecto mais privado da autoconsciência foram identificadas por Burnkrant e Page (1984): autorreflexão (do original "self-reflection") e consciência de estados internos (do original "internal state awareness"). Enquanto o primeiro fator mensuraria a tendência de refletir sobre si ou a autorreflexividade, o segundo determinaria o nível de consciência do indivíduo em relação ao que ocorre internamente em seu corpo, como a capacidade de perceber os batimentos cardíacos e a respiração, além de sensações como euforia, tranquilidade, depressão e raiva.

A despeito de encontrarem resultados similares a esses, Anderson, Bohon e Berrigan (1986) discordaram da nomeação dos fatores e indicaram os termos autoopressão (do original "self-oppression") e consciência equilibrada ou imparcial de si (do original, "balanced self-awareness") em substituição a autorreflexão ("selfreflection") e consciência de estados internos ("internal state awareness"), nessa ordem. Conceitualmente, os termos propostos por Anderson et al. ressaltam um estilo negativo da autorreflexão, caracterizado por pensamentos ruminativos e por uma desconfiança em relação a si mesmo; e outro mais positivo ou neutro da consciência de estados internos, que expressaria um interesse ou autofoco atencional benéficos à compreensão das próprias emoções e estados corporais.

Mais tarde, o modelo bidimensional da autoconsciência privada foi reforçado nos trabalhos de Creed e Funder (1998) e Trapnell e Campbell (1999). Para os primeiros pesquisadores, contudo, autorreflexão ("self-reflection") e consciência de estados internos ("internal state awareness") não estariam em pólos opostos, mas expressariam, ambos, uma tendência à introspecção. A diferença residiria no fato de que indivíduos mais conscientes de seu estado interno pareciam possuir um estilo de personalidade mais resolutivo e socialmente funcional quando comparados 
com os predominantemente autorreflexivos, que costumavam sentir culpa, ansiedade e medo de serem avaliados por outros.

O debate sobre os efeitos mal-adaptativos da autorreflexão ganhou destaque no final da década de 1990, quando Trapnell e Campbell (1999) sugeriram que a autoconsciência seria determinada não apenas pela direção do foco atencional, mas também pelas motivações desse funcionamento. Essas motivações poderiam ser definidas tanto por um elevado grau de neuroticismo, sustentado por pensamentos e sentimentos negativos de ameaça, perda e injustiça (perfil ruminativo), quanto por uma curiosidade genuína em ampliar o autoconhecimento de maneira criativa e exploratória (perfil reflexivo) (Trapnell \& Campbell, 1999; Zanon \& Teixeira, 2006). Tal compreensão é a base para o seu Questionário de Ruminação e Reflexão (QRR) (Trapnell \& Campbell, 1999).

A dimensão consciência de estados internos ("internal state awareness") retornou ao debate sob a denominação "insight", por meio do trabalho de Grant, Franklin e Langford (2002). Com o objetivo de auxiliar pesquisadores e profissionais na avaliação de suas práticas e intervenções clínicas e investigar as características adaptativas e construtivas da autoconsciência que poderiam favorecer mudanças comportamentais, propuseram a Escala de Autorreflexão e "Insight" (EAI) que, como o próprio nome especifica, abrange os fatores autorreflexão e "insight".

Na perspectiva defendida por Grant et al. (2002), a autoconsciência privada seria um importante componente do processo metacognitivo conhecido em inglês como "psychological mindedness". De difícil tradução para o português, a expressão pode ser entendida como uma propensão ou predisposição (envolve, portanto, habilidade e motivação) em engajar-se emocional e intelectualmente na investigação de como e por que o próprio indivíduo e outros se comportam, pensam e sentem da maneira como fazem (Grant, 2001). A metacognição é, por sua vez, um processo executivo que envolve conhecer, monitorar e controlar quaisquer aspectos da cognição (Lai, 2011; Schraw \& Moshman, 1995), a fim de regular o processamento de informações e o comportamento (Koriat, 2006). Mais especificamente, implica conhecer os processos e as competências - próprias e gerais - requeridos na execução de tarefas (o que, quando e por que); planejar (definir metas, escolher estratégias e alocar recursos de maneira adequada); monitorar e avaliar periodicamente o desempenho e o conhecimento adquirido no processo; e controlar toda a operação, regulando esforços e o uso de recursos quando necessário (Koriat, 2006; Lai, 2011; Schraw \& Moshman, 1995).
Dentro desse contexto, pode-se entender que autorreflexão e "insight" são demandados nas fases de monitoramento e avaliação, respectivamente, de modo que o indivíduo seja capaz de ajustar as estratégias por ele empregadas (Grant et al., 2002). A autorreflexão responderia pelo monitoramento dos próprios pensamentos, sentimentos e comportamentos; enquanto o "insight", pela habilidade de compreendê-los, avaliálos sob diferentes e novas perspectivas e expressálos (Grant et al., 2002), a fim de tecer julgamentos e autorregular-se (Hays et al., 2002; Stein \& Grant, 2014). A concepção de "insight" utilizada por Grant et al. (2002), diferentemente da concepção cognitivista difundida pela expressão o "momento Aha" (Stein \& Grant, 2014), não envolve uma reação emocional ou um sentimento súbito do indivíduo em relação à solução de um problema (Kounios \& Beeman, 2009). Trata-se, por outro lado, de uma tendência ou característica do indivíduo de, ao direcionar-se propositadamente a uma mudança, entender com clareza aspectos de si mesmo e das situações que a ele se apresentam (Stein \& Grant, 2014).

Ainda que integrem um mesmo processo, autorreflexão e "insight" seriam, para Grant et al. (2002), dimensões independentes, visto que um indivíduo pode dedicar tempo refletindo sobre si (autorreflexão) sem, necessariamente, alcançar um entendimento esclarecedor sobre a sua condição ou experiência ("insight"). Como consequência, pessoas com alto nível de autorreflexão, porém baixo de "insight", podem apresentar ou manter pensamentos e atitudes prejudiciais ao seu equilíbrio psicológico e social, como a autorruminação (Grant, 2001; Grant et al., 2002). Logo, a efetividade da autorregulação e o aprendizado, que poderiam culminar com uma mudança comportamental, dependeriam tanto de motivação e de uma boa habilidade de automonitoramento (demanda autorreflexão) quanto de autoavaliação (vinculada ao “insight") (Grant et al., 2002).

Segundo Grant et al. (2002), os 20 itens que compõem a EAI são capazes de abranger os três domínios da experiência humana: pensamentos, sentimentos e comportamentos. Desses, 12 avaliam os aspectos "engajamento na reflexão" e "necessidade por reflexão", elementos da dimensão autorreflexão, como em "Eu raramente despendo tempo na reflexão sobre mim", "É importante para mim tentar entender o que significam meus sentimentos"; e oito itens avaliam a dimensão "insight", expressa em afirmações como "Eu normalmente tenho uma ideia bem clara sobre por que tenho me comportado de uma certa maneira".

Apesar da histórica polêmica em torno da autoconsciência privada e da possibilidade de mensurá-la, 
que não se encerra com as proposições de Grant et al. (2002), parece não haver, até o momento, revisões sistemáticas que, além de organizarem o corpo de evidências sobre o tema, auxiliariam na compreensão do fenômeno e no consequente avanço da área. A fim de atender, parcialmente, a essa lacuna, este trabalho objetivou verificar como pesquisas recentes apresentam a interação das dimensões autorreflexão e "insight" com a cognição e o comportamento a partir de estudos empíricos que utilizaram a EAI como instrumento de avaliação psicológica.

\section{Método}

\section{Fontes de informação e estratégia de pesquisa}

O levantamento bibliográfico foi realizado em janeiro de 2017, a partir das bases de dados disponíveis no Portal de Periódicos da Coordenação de Aperfeiçoamento de Pessoal de Nível Superior CAPES, por meio dos termos "self-consciousness"; "self-awareness"; "self-reflection"; "insight"; SRIS; autoconsciência; autorreflexão; EAI; combinados conforme segue: ("self-reflection" AND insight) SRIS; autoconsciência AND ((autorreflexão AND insight) EAI); "self-consciousness" AND (("self-reflection" AND insight) SRIS); "self-awareness" AND (("self- reflection" AND insight) SRIS); ("self-consciousness" OR "self-awareness") AND (("self-reflection" AND insight) SRIS). O período de publicação foi definido como sendo de 2002 a 2017, pelo fato de a EAI, instrumento foco desta revisão, ter sido lançada em 2002.

\section{Critérios de seleção e análise dos estudos}

Foram considerados os artigos científicos revisados por pares, disponíveis eletrônica e gratuitamente, nos idiomas inglês e português. Após análise de título e resumo e excluídas as duplicidades, a seção método auxiliou a seleção das publicações cujo título e resumo não foram suficientes para a avaliação de sua elegibilidade. Os estudos que utilizaram a EAI apenas parcialmente, restringindo o número de itens da escala $(n=1)$ ou analisando apenas uma das dimensões de interesse (autorreflexão ou "insight") $(\mathrm{n}=6)$, foram excluídos. Visando a uma maior uniformidade na comparação dos dados e tendo em conta que a EAI não foi criada para mensurar a atividade autoconsciente patológica (DaSilveira et al., 2012), um estudo $(n=1)$ com essa abordagem também foi excluído. A Figura 1 ilustra o processo de identificação e triagem dos estudos a partir dos critérios de elegibilidade definidos.

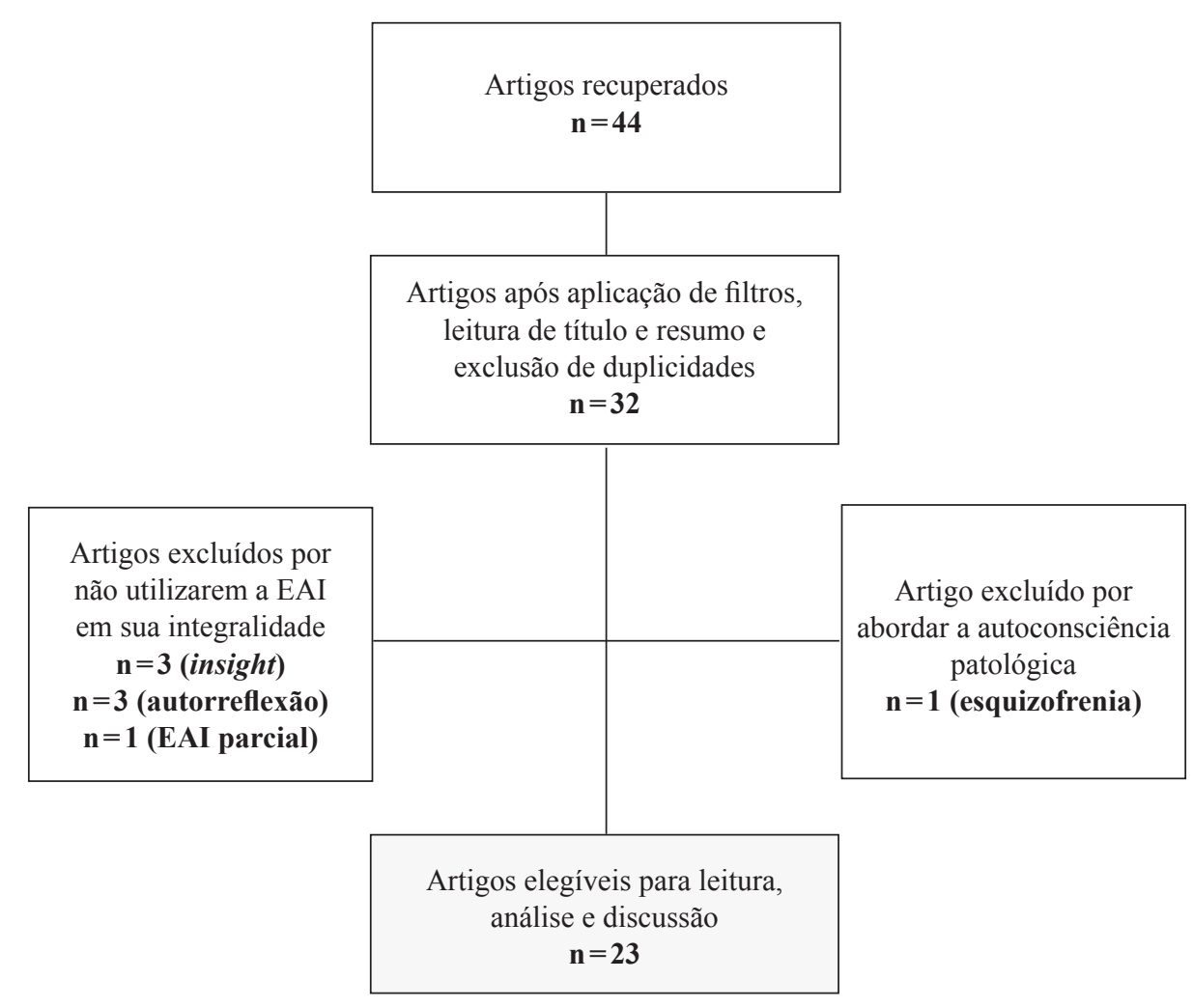

Figura 1. Processo de identificação e triagem de artigos para análise. 
Dos 31 artigos identificados, inicialmente, para leitura de texto completo, 23 foram selecionados como objeto de categorização, análise e discussão. Os dados foram coletados e analisados de acordo com as seguintes categorias: ano de publicação; país de origem; método; área de conhecimento; variáveis estudadas; entendimento dos autores em relação à autoconsciência, autorreflexão e "insight"; e principais resultados.

\section{Resultados}

Um resumo dos resultados das categorias analisadas é apresentado na Tabela 1 e as principais associações entre as variáveis investigadas nos estudos revisados são reportadas mais adiante.

No que diz respeito ao entendimento dos autores em relação à autoconsciência, autorreflexão e "insight", a maioria dos estudos concentra sua atenção na análise

TABELA 1

Caracterização de estudos publicados entre 2002 e 2017 que utilizaram a EAI.

\begin{tabular}{|c|c|c|c|c|c|c|}
\hline Ano & Autores & Pais & Área & Método & Likert & $\begin{array}{l}\text { Construtos avaliados além de } \\
\text { Autorreflexão e Insight }\end{array}$ \\
\hline 2002 & $\begin{array}{l}\text { Grant, A. M., Franklin, J., } \\
\text { \& Langford, P. }\end{array}$ & Austrália & Psicologia & Quantitativo & 6 & $\begin{array}{l}\text { Alexitimia; Depressão; Ansiedade; Estresse; } \\
\text { Flexibilidade cognitiva; Autocontrole }\end{array}$ \\
\hline 2003 & Grant, A. M & Austrália & Psicologia & Qualitativo & Não específica & Depressão; Estresse; Ansiedade \\
\hline 2008 & Roberts. C. \& Stark, P. & $\begin{array}{l}\text { Austrália e } \\
\text { Reino Unido }\end{array}$ & Medicina & Quantitativo & 5 & $\begin{array}{l}\text { Autorregulação; Desempenho acadêmico- } \\
\text { profissional }\end{array}$ \\
\hline 2009 & Butler, J.C. & EUA & Psicologia & Quantitativo & 6 & Autoritarismo; Medo \\
\hline 2009 & $\begin{array}{l}\text { Haga, S. M., Kraft, P., \& } \\
\text { Corby, E.K. }\end{array}$ & $\begin{array}{l}\text { Austrália, EUA e } \\
\text { Noruega }\end{array}$ & Psicologia & Quantitativo & 6 & $\begin{array}{l}\text { Autorregulação; Extroversão; Neuroticismo; } \\
\text { Depressão; Satisfação com a vida; Afeto } \\
\text { positivo e negativo }\end{array}$ \\
\hline 2009 & Lyke, J.A. & EUA & Psicologia & Quantitativo & 6 & $\begin{array}{l}\text { Felicidade subjetiva; Satisfação com a vida; } \\
\text { Depressão; Ansiedade }\end{array}$ \\
\hline 2010 & Gerace, A. \& Day, A. & Austrália & Psicologia & Qualitativo & 5 & $\begin{array}{l}\text { Empatia; Perdão; Ruminação e } \\
\text { Reflexão (QRR); Propensão à mudança; } \\
\text { Adaptabilidade social; Adesão ao programa }\end{array}$ \\
\hline 2010 & $\begin{array}{l}\text { Richards, K. C., Campenni, C.E., \& } \\
\text { Muse-Burke, J.L. }\end{array}$ & EUA & Psicologia & Quantitativo & 6 & $\begin{array}{l}\text { Autocuidado; Bem-estar subjetivo; } \\
\text { Mindfulness }\end{array}$ \\
\hline 2010 & $\begin{array}{l}\text { Sauter, F.M., Heyne, D., } \\
\text { Blöte, A. W., van Widenfelt, B. M., } \\
\text { \& Westenberg, P.M. }\end{array}$ & Holanda & Psicologia & Quantitativo & 6 & Desempenho cognitivo \\
\hline 2011 & Harrington, R. \& Loffredo, D.A. & EUA & Psicologia & Quantitativo & 6 & $\begin{array}{l}\text { Bem-estar subjetivo; Ruminação e Reflexão } \\
\text { (QRR); Ansiedade social; Autoconsciência } \\
\text { pública e privada; Satisfação com a vida }\end{array}$ \\
\hline 2011 & Silvia, P. J. \& Phillips, A. G. & EUA & Psicologia & Quantitativo & 7 & $\begin{array}{l}\text { Ruminação e Reflexão (QRR); } \\
\text { Autoconsciência pública e privada; } \\
\text { Depressão; Ansiedade; Afeto positivo e } \\
\text { negativo; Autoestima }\end{array}$ \\
\hline 2011 & $\mathrm{Xu}, \mathrm{X}$. & EUA & Psicologia & Misto & 6 & Desempenho cognitivo \\
\hline 2012 & Chung, S.-H., Su, Y.-F., \& Su, S.-W. & Taiwan & Psicologia & Quantitativo & 6 & $\begin{array}{l}\text { Flexibilidade cognitiva; Resistência à } \\
\text { mudança }\end{array}$ \\
\hline 2012 & $\begin{array}{l}\text { DaSilveira, A., DeCastro, T.G., \& } \\
\text { Gomes, W. B. }\end{array}$ & Brasil & Psicologia & Quantitativo & 5 & $\begin{array}{l}\text { Ruminação e Reflexão (QRR); Ansiedade } \\
\text { social; Autoconsciência pública e privada; } \\
\text { Autoabsorção }\end{array}$ \\
\hline 2013 & Carr, S.E. \& Johnson, P.H. & Austrália & Medicina & Quantitativo & 5 & Desempenho acadêmico \\
\hline 2014 & van Dulmen et al. & Holanda & Fisioterapia & Qualitativo & 5 & Desempenho profissional \\
\hline 2015 & $\begin{array}{l}\text { DaSilveira, A., DeSouza, M.L., \& } \\
\text { Gomes, W.B. }\end{array}$ & Brasil & Psicologia & Quantitativo & 5 & $\begin{array}{l}\text { Ruminação e Reflexão (QRR); Ansiedade } \\
\text { social; Autoconsciência pública e privada; } \\
\text { Autoabsorção; Mindfulness }\end{array}$ \\
\hline 2015 & Eng, C.-J. \& Pai, H.-C. & Taiwan & Enfermagem & Quantitativo & 5 & $\begin{array}{l}\text { Estresse; Autorregulação; Desempenho } \\
\text { acadêmico-profissional }\end{array}$ \\
\hline 2015 & Mass, M.J.M. et al. & Holanda & Fisioterapia & Qualitativo & Não específica & Desempenho profissional \\
\hline 2015 & Pai, H.-C. & Taiwan & Enfermagem & Misto & 5 & $\begin{array}{l}\text { Estresse; Desempenho acadêmico } \\
\text { profissional; Qualidade do ensino }\end{array}$ \\
\hline 2016 & Cowden, R. G. \& Meyer-Weitz, A. & África do Sul & Psicologia & Quantitativo & 6 & Estresse; Resiliência \\
\hline 2016 & $\begin{array}{l}\text { Harrington, R., Loffredo, D.A., \& } \\
\text { Perz, C.A. }\end{array}$ & EUA & Psicologia & Quantitativo & Não específica & $\begin{array}{l}\text { Mindfulness; Satisfação com a vida; } \\
\text { Felicidade subjetiva }\end{array}$ \\
\hline 2016 & Sutton, A. & Reino Unido & Psicologia & Misto & 6 & Mindfulness; Ruminação e Reflexão (QRR) \\
\hline
\end{tabular}


de uma possível associação entre as dimensões da autoconsciência privada e as variáveis de interesse, e não propõe ou se aprofunda em discussões conceituais e teóricas sobre os construtos. Entre os pesquisadores que procuram explicitar sua compreensão sobre os fenômenos estão DaSilveira, DeSouza e Gomes (2015) e Richards, Campenni e Muse-Burke (2010).

DaSilveira et al. (2015) discorrem sobre a relação entre os conceitos de autorreflexão, "awareness" e "insight". Orientados pelos resultados alcançados na pesquisa que, em linhas gerais, visava a verificar os fatores comuns que as escalas de autoconsciência disposicional se propunham a mensurar, eles sugerem uma distinção epistemológica no conceito de autoconsciência que poderia ser evidenciada nas definições de autorreflexão e "awareness". Enquanto o primeiro construto (autorreflexão) compreenderia uma cognição processual que envolve pensamento e memória, o segundo ("awareness") expressaria uma cognição fenomênica, "incorporada" ou "situada" (p.3). Explicando de outro modo, o termo "autorreflexão" abrangeria experiências passadas e julgamentos de conteúdos já internalizados, ou seja, processados por meio de pensamentos e fala interna; e o termo "awareness" compreenderia experiências do tempo presente ainda não racionalizadas pelo indivíduo, um conceito que expressaria, sob a perspectiva fenomenológica, a "autoconsciência préreflexiva" (p.10). No que tange ao "insight", pelo fato de correlacionar-se negativamente com variáveis mal-adaptativas, comportando-se de maneira oposta à de construtos relacionados à autorreflexividade e similar a de "awareness", DaSilveira et al. advertem sobre a necessidade de novas pesquisas que evidenciem espaços de convergência e/ou divergência entre eles.

Richards et al. (2010) empregam o termo "selfawareness" para referirem-se à autoconsciência e tal como propuseram Fenigstein et al. (1975), entendem-na como um estado. Criticam a "escassa e desatualizada" (p. 250) literatura dedicada às discussões conceituais sobre o fenômeno da autoconsciência e mencionam a similaridade semântica entre "self-awareness", "selfconsciousness", "insight" e "mindfulness" presente nas publicações da área. Apesar das semelhanças, citando outros autores, Richards et al. defendem que haveria uma sutil diferença entre "self-awareness" e "mindfulness": o primeiro conceito envolveria uma consciência interna das próprias cognições e emoções; o segundo, além de uma consciência interna ou do que lhe é próprio, uma consciência externa ou do ambiente circundante. Para eles, quando posto em prática, o "mindfulness" pode favorecer o "insight" e promover o aumento do que entendem por "self-awareness" (Richards et al., 2010).

No tocante a associações entre as duas dimensões da autoconsciência privada propostas por Grant et al. (2002) e outras variáveis, os estudos revisados convergiram para uma relação positiva entre "insight" e reações emocionais, cognitivas e comportamentais saudáveis e adaptativas, porém divergiram sobre os benefícios da autorreflexão na autorregulação. Grant et al. (2002) argumentam que o processo de autorreflexão pode se apresentar de diferentes maneiras a depender do indivíduo e não pressupõe, necessariamente, o desenvolvimento do "insight". Enquanto para alguns pode favorecer o aprendizado, o desempenho ou a competência profissional (Cowden \& Meyer-Weitz, 2016; Eng \& Pai, 2015; Pai, 2015), e o aperfeiçoamento do "insight" (Pai, 2015), para indivíduos com tendência à ansiedade, refletir sobre si mesmo, seus pensamentos, sentimentos e comportamentos, pode demandar um esforço consciente extra e revelar um processo disfuncional (Grant et al., 2002).

Fazendo um comparativo com o modelo de "coping" de Lazarus e Folkman (Antoniazzi, A. S., Dell'Aglio, D. D., \& Bandeira, D. R., 1998), Grant et al. (2002) consideram a existência de pelo menos dois tipos de autorreflexão: um estilo reflexivo que denominaram autorreflexão focada na solução de problemas e atingimento de metas (do original "problem-solving self-reflection"); e outro que designaram reflexão focada em si ou reflexão sobre si (do original "selffocused self-reflection"), que enfatiza o entendimento e o gerenciamento de reações emocionais, cognitivas e comportamentais negativas. Ambos os estilos seriam empregados por um mesmo indivíduo, apesar de haver uma tendência de preferir um ao outro (Grant et al., 2002).

De forma predominante, os resultados dos estudos evidenciam uma associação positiva entre autorreflexão e ruminação (Sauter, Heyne, Blöte, van Widenfelt, \& Westenberg, 2010; Silvia \& Phillips, 2011); autorreflexão, afeto negativo e depressão (Silvia \& Phillips, 2011); autorreflexão e ansiedade (Grant et al., 2002); autorreflexão e estresse (Grant et al., 2002; Silvia \& Phillips, 2011); autorreflexão e autoabsorção privada (DaSilveira et al., 2012); autorreflexão e resiliência (Cowden \& Meyer-Weitz, 2016). Por outro lado, apontam uma associação negativa entre "insight" e ruminação (DaSilveira et al., 2012, 2015); “insight" e internalização de problemas (Sauter et al., 2010); "insight" e alexitimia (Grant et al., 2002); "insight" e estresse (Cowden \& Meyer-Weitz, 2016; Grant et al., 2002); "insight", depressão e ansiedade (Grant et al., 2002; Silvia \& Phillips, 2011); "insight”, autoabsorção 
pública e autoabsorção privada (DaSilveira et al., 2012).

Roberts e Stark (2008) sugerem que altos níveis de engajamento na reflexão podem revelar um processo ruminativo e impedir que o indivíduo alcance o "insight", importante na solução de problemas. A esse entendimento, DaSilveira et al. (2012) acrescentam que também a autoabsorção, considerada um "subproduto mal-adaptativo da autorreflexão" (p. 161), "drena" recursos cognitivos podendo acarretar uma série de consequências negativas, como a diminuição do bemestar subjetivo.

De maneira análoga, a intensidade da necessidade por reflexão influencia a demanda de recursos mentais, podendo causar diferentes efeitos a depender do que a situação ou a tarefa requeira. Os resultados de um experimento com universitários envolvendo tarefas de linguagem $(\mathrm{Xu}, 2011)$ denotam uma associação entre altos níveis de necessidade por reflexão e baixos níveis de proeficiência em vocabulário, porém menor incidência de erros de intrusão em tarefas de reconhecimento. Indivíduos com altos níveis de "insight", por sua vez, apresentam maior acurácia e rapidez em suas respostas (Xu, 2011).

A presença de "insight" também parece atuar na flexibilidade cognitiva e autorregulação (Chung, Su, \& Su, 2012; Grant et al., 2002; Silvia \& Phillips, 2011); na "performance" esportiva (Cowden \& Meyer-Weitz, 2016); na redução de afeto negativo; nos níveis de depressão e ansiedade; na elevação de afeto positivo e autoestima (Silvia \& Phillips, 2011); e no sentimento positivo de bem-estar psicológico (Harrington \& Loffredo, 2011); de satisfação com a vida (Harrington \& Loffredo, 2011; Lyke, 2009); e de felicidade subjetiva (Harrington, Loffredo, \& Perz, 2016; Lyke, 2009). Contudo, somente os indivíduos que apresentam níveis mais altos de "insight" alegam experienciar sentimentos de felicidade e de satisfação com a vida significativos, ao passo que o grau de autorreflexão parece não exercer qualquer influência nessa equação, que pode ainda ser afetada por diferenças culturais (Lyke, 2009).

Em contextos de desenvolvimento profissional, estudos verificaram ainda que o aumento de autoconsciência pode facilitar o entendimento do próprio estresse e da ansiedade e influenciar positivamente o aprendizado e o desempenho (Cowden \& Meyer-Weitz, 2016; Eng \& Pai, 2015; Pai, 2015). No caso de atletas, Cowden e Meyer-Weitz (2016) indicam que autorreflexão e "insight" atuam nos níveis de resiliência, apesar de o "insight" maximizar essa relação, favorecendo a adaptabilidade em cenários desfavoráveis e o otimismo em relação ao futuro.
Esportistas que possuem um claro entendimento de seus pensamentos, sentimentos e comportamentos parecem lidar melhor com as próprias emoções e ser capazes de ajustar reações a fim de reduzir o estresse, superar adversidades e atingir suas metas. Logo, rotinas que auxiliem a autorreflexão, como a prática de escrever ou falar sobre experiências, acompanhadas por uma abordagem estruturada de identificação e avaliação das fontes de estresse e suas possíveis consequências, podem ser estratégias eficazes para a autorregulação e, consequentemente, para a "performance" esportiva (Cowden \& Meyer-Weitz, 2016).

Conclusões similares foram alcançadas por pesquisas envolvendo acadêmicos de enfermagem (Eng \& Pai, 2015; Pai, 2015). Os dados revelam que o estresse resultante da prática clínica se reduz quando autorreflexão e "insight" aumentam, e que programas para desenvolvimento de habilidades autorreflexivas e de enfrentamento ainda na academia podem aprimorar a atuação dos futuros profissionais. Os benefícios da autoconsciência também foram atestados pelos resultados de Richards et al. (2010), que apontam uma correlação positiva entre "self-awareness", bem-estar e a importância de autocuidar-se em especialistas da saúde mental, com o "mindfulness" mediando essa relação.

Dentro de um cenário organizacional em mudança, Chung, Su e Su (2012) confirmam Grant et al. (2002) na correlação positiva entre "insight" e flexibilidade cognitiva e sugerem a autorreflexão como variável antecedente à flexibilidade cognitiva. Essa, por sua vez, estaria negativamente associada à resistência afetiva, resistência cognitiva e resistência comportamental (três variáveis da resistência organizacional). Dito de outro modo, trabalhadores com boa flexibilidade cognitiva mostram-se mais propensos a inibir sentimentos e pensamentos negativos e a aceitar mudanças no ambiente profissional. Indiretamente, os autores concluem que autorreflexão e "insight" variam em direção oposta à resistência organizacional, e recomendam que em situações de recrutamento, as empresas se atentem para os níveis de autorreflexão e “insight" dos candidatos (Chung et al., 2012).

Para alguns pesquisadores, idade e gênero podem influenciar a autoconsciência privada, apesar de essa relação não ser consensual. Enquanto Roberts e Stark (2008) e Haga, Kraft e Corby (2009) apontam para uma correlação positiva entre "insight" e idade, Sauter, Heyne, Blöte, van Widenfelt e Westenberg (2010) sugerem que a autorreflexão pode se intensificar na adolescência, entre 14 e 18 anos, e não obedecer a uma progressão linear. Ao contrário de Haga et al. (2009), para os quais autorreflexão e "insight" 
são características de personalidade "maleáveis" (p.288) que tendem a crescer com o avançar da idade e configuram importantes aspectos da regulação emocional saudável, para Sauter et al. (2010), mais do que uma capacidade que amadurece com o tempo, o "insight" parece tratar-se de uma disposição individual cujo desenvolvimento estaria dissociado do desenvolvimento da autorreflexão.

No que se refere ao gênero, escores superiores de "insight" foram verificados em homens (Roberts \& Stark, 2008) e de autorreflexão, em mulheres (DaSilveira et al., 2015; Sauter et al., 2010). Diferenças de gênero também foram constatadas em estudo que analisou a influência da autoconsciência privada no uso da reavaliação cognitiva e da supressão expressiva como estratégias de regulação emocional (Haga et al., 2009). Os resultados mostraram que em mulheres, a autorreflexão parece desempenhar um papel importante na evitação da supressão e no incremento da reavaliação cognitiva, enquanto o "insight" reduz o uso da supressão. Já em homens, ambas as dimensões da autoconsciência privada influenciam o aumento da reavaliação cognitiva, mas não provocam qualquer efeito na estratégia de supressão.

Fatores como o nível de engajamento no processo de autorrefletir, os mecanismos psicológicos e comportamentais envolvidos ou empregados nesse processo, e as razões que levam o indivíduo a se engajar também podem afetar a relação entre autorreflexão, "insight", comportamento e desempenho, alegam Grant et al. (2002). Em estudo que avaliou o impacto de um programa de "coaching" de vida em universitários (Grant, 2003), verificou-se que após intervenção, níveis de autorreflexão diminuíram ao passo que os de "insight" aumentaram, sugerindo que no ciclo de autorregulação, indivíduos tendem a se engajar menos no processo autorreflexivo e experienciar maior "insight", movimento que, de modo geral, favoreceria o desenvolvimento e a mudança.

\section{Discussão}

Esta revisão sistemática teve como objetivo verificar a relação entre a autorreflexão e o "insight", dimensões da autoconsciência privada, na cognição e no comportamento, por meio da análise de estudos que fizeram uso da Escala de Autorreflexão e "Insight" (EAI), idealizada por Grant et al. (2002). A mensuração, o controle e o aprimoramento desse elo têm atraído pesquisadores principalmente da área da saúde, tendo em vista que o uso inadequado de recursos envolvidos no processo de refletir sobre os próprios pensamentos, sentimentos e comportamentos, entendê-los, avaliá-los e modificá-los, pode acarretar prejuízos ao desempenho cognitivo, ao bem-estar físico e psicológico, e às interações sociais. Experiência vivenciada tanto por profissionais em formação ou no exercício de sua atividade (Carr \& Johnson, 2013; Cowden \& MeyerWeitz, 2016; Eng \& Pai, 2015; Mass et al., 2015; Pai, 2015; Richards et al., 2010; Roberts \& Stark, 2008; van Dulmen et al., 2014), quanto por aqueles que estão sob seus cuidados e atenção (Butler, 2009; Chung et al., 2012; DaSilveira et al., 2012, 2015; Gerace \& Day, 2010; Grant, 2003; Grant et al., 2002; Haga et al., 2009; Harrington \& Loffredo, 2011; Harrington et al., 2016; Lyke, 2009; Sauter et al., 2010; Silvia \& Phillips, 2011; Sutton, 2016; Xu, 2011).

Os resultados dos estudos analisados convergiram para uma relação positiva entre "insight" e reações emocionais, cognitivas e comportamentais saudáveis e adaptativas, porém divergiram sobre os benefícios da autorreflexão para a autorregulação. Em alguns indivíduos, os níveis de autorreflexividade variam na mesma direção em que os de ruminação (Sauter et al., 2010; Silvia \& Phillips, 2011), ansiedade (Grant et al., 2002), depressão e afeto negativo (Silvia \& Phillips, 2011), contribuindo para o desequilíbrio psíquico; enquanto em outros, atuam favorecendo o aprendizado, o exercício profissional (Eng \& Pai, 2015; Pai, 2015) e a resiliência (Cowden \& Meyer-Weitz, 2016).

A influência de gênero e idade na autoconsciência privada também divergiu entre os autores revisados, de modo a não ser possível tecer conclusões a respeito. Contudo, no que se refere à idade, considerando que autorreflexão e "insight" são elementos do processo metacognitivo (Grant et al., 2002) e que o desenvolvimento da cognição ocorre ao longo da vida em função do amadurecimento biológico, de experiências pessoais e de interações com o meio, tende-se a assumir como mais prováveis de ocorrerem em adultos do que em adolescentes ou jovens, associações entre níveis de autoconsciência privada (com predomínio para o "insight") e respostas adaptativas ou benéficas ao equilíbrio do indivíduo, como reportam Haga et al. (2009) e Roberts e Stark (2008).

Em alguns aspectos, as discordâncias encontradas parecem refletir uma polêmica que sempre permeou as discussões em torno das dimensões que compõem o fenômeno da autoconsciência e que deu origem a diversas explicações, nomenclaturas e instrumentos psicométricos como a EAC (Fenigstein et al., 1975), o QRR (Trapnell \& Campbell, 1999) e a EAI (Grant et al., 2002). Alvo de crítica de pesquisadores (Richards et al., 2010), a diversidade terminológica para expressar um único, porém complexo fenômeno, parece retardar o avanço da área e dificultar o entendimento e o uso, 
principalmente por parte de cientistas cuja língua nativa não seja o inglês.

Termos como "consciousness", "awareness", "self", "self-consciousness", "self-awareness", "self-reflection" e derivações, aos quais parecem ser atribuídos significados distintos, por vezes sutis, podem ter o sentido distorcido quando empregados por autores estrangeiros, situação que se agrava em traduções. Tal fato torna ainda mais importante e necessária a menção clara da fundamentação e do direcionamento teóricos dos autores, o que não se verifica na maioria dos estudos revisados.

De maneira geral, é possível sugerir que a autoconsciência privada indica uma tendência à introspecção (Creed \& Funder, 1998; Fenigstein et al., 1975) que pode se manifestar em maior ou menor grau a depender do indivíduo, de sua trajetória de vida (Fenigstein et al., 1975; Grant et al., 2002) e até mesmo de influências culturais (Lyke, 2009). O modo como cada um percebe e expressa pensamentos, emoções e comportamentos e se relaciona consigo - e também com o mundo - atende a diversas finalidades (Snyder, 1974) e motivações (Grant el al., 2002; Trapnell \& Campbell, 1999) e pode ser atribuído a aspectos disposicionais e situacionais (Fenigstein et al., 1975). Trata-se de um processo interativo, dinâmico e intencional, que se materializa no corpo, a partir de vivências, relações sócio-históricas, cultura, afetividade, diálogo e expressão.

Independente da nomenclatura ou da explicação que se dê para o fenômeno e para os fatores que o compõem, a autoconsciência privada pressupõe uma dimensão reflexiva que parece preceder escolhas e retroalimentar o processo, tornando-o mais ou menos favorável ao equilíbrio psíquico e social. Gastos excessivos de tempo e de recursos cognitivos e emocionais nessa etapa prejudicam o desempenho $(\mathrm{Xu}$, 2011) e podem interromper o avanço do indivíduo no ciclo autorregulatório (DaSilveira et al., 2012; Grant et al., 2002; Roberts \& Stark, 2008). Logo, deduz-se que apesar de desencadear o processo, a autorreflexividade por si só não é suficiente, sendo o "insight" elemento fundamental e decisivo para alcançar o que se almeja ou evitar o indesejável (Cowden \& Meyer-Weitz, 2016; Grant et al., 2002).

A partir da literatura revisada também é viável indicar que a autoconsciência, aqui entendida como a capacidade de reconhecer, compreender, aperfeiçoar, inovar e sistematizar processos cognitivos, antevendo consequências e soluções, pode ser aprimorada. Programas ou intervenções que desenvolvam a autorreflexão e o "insight" podem favorecer a mudança comportamental e o aprendizado e beneficiar o indivíduo (Cowden \& Meyer-Weitz, 2016; Eng \& Pai, 2015; Grant, 2003; Grant et al., 2002; Pai, 2015).

Ainda não foram estabelecidos parâmetros a partir dos quais se poderia classificar níveis de autoconsciência privada, em suas dimensões autorreflexão e "insight", como benéficos ou prejudiciais à saúde dos indivíduos. No entanto, resultados de pesquisas (Grant, 2003; Grant et al., 2002; DaSilveira et al., 2012; Lyke, 2009; Roberts \& Stark, 2008; Xu, 2011) sugerem que esse limiar existe. Assim como ocorre com outras variáveis psicológicas mensuráveis, como o estresse, a variação da autoconsciência privada parece obedecer a uma curva em forma de sino na qual as extremidades ou caudas podem refletir dificuldades de adaptação ou de manejo cognitivo e comportamental, representando áreas de risco.

Número maior de estudos quantitativos que apliquem a EAI em contextos e populações variadas seria necessário para que parâmetros e conclusões dessa natureza fossem instituídos. Estudos qualitativos e experimentais também seriam úteis na medida em que poderiam contribuir com um entendimento mais aprofundado do fenômeno e, nesse sentido, com as discussões conceituais. Destaque aí para as pesquisas com neuroimagem cujas técnicas têm permitido investigar com maior precisão temporal e espacial, informações comportamentais antes acessíveis apenas por autorrelato.

Por fim, é necessário considerar que, em função dos filtros de busca estabelecidos, esta revisão apresentou apenas um recorte dos estudos da autoconsciência privada, concentrando sua atenção nas dimensões autorreflexão e "insight" mensuradas pela EAI. Novos estudos e revisões, com diferentes métodos, ênfases e abrangências, certamente auxiliariam o acesso, a compreensão e o aumento do interesse de outros pesquisadores a uma temática originalmente complexa e controversa, mas com potencial para esclarecer uma importante dimensão do funcionamento psicológico humano. 


\section{Referências}

Anderson, E. M., Bohon, L. M., \& Berrigan, L. P. (1996). Factor Structure of the Private Self-Consciousness Scale. Journal of Personality Assessment, 66(1), 144-152. https://doi.org/10.1207/s15327752jpa6601_11

Antoniazzi, A. S., Dell'Aglio, D. D., \& Bandeira, D. R. (1998). O conceito de coping: uma revisão teórica. Estudos de Psicologia, 3(2), 273-294. https://doi.org/10.1590/S1413-294X1998000200006

Burnkrant, R. E. \& Page, T. J. Jr. (1984). A modification of the Fenigstein, Scheier, and Buss Self-Consciousness Scales. Journal of Personality Assessment, 48(6), 629-637. https://doi.org/10.1207/s15327752jpa4806_10

Butler, J. C. (2009). Authoritarianism and Fear of Deviance. North American Journal of Psychology, 11(1), 49-64.

Carr, S. E. \& Johnson, P. H. (2013). Does self-reflection and insight correlate with academic performance in medical students?. BMC Medical Education, 13(113), 1-5. https://doi.org/10.1186/1472-6920-13-113

Chung, S.-H., Su, Y.-F., \& Su, S.-W. (2012). The impact of cognitive flexibility on resistance to organizational change. Social Behavior and Personality, 40(5), 735-746. https://doi.org/10.2224/sbp.2012.40.5.735

Cowden, R. G. \& Meyer-Weitz, A. (2016). Self-reflection and self-insight predict resilience and stress in competitive tennis. Social Behavior and Personality, 44(7), 1133-1150. https://doi.org/10.2224/sbp.2016.44.7.1133

Creed, A. T. \& Funder, D. C. (1998). The Two Faces of Private Self-Consciousness: Self Report, Peer-Report, and Behavioral Correlates. European Journal of Personality, 12(6), 411-431. https://doi.org/10.1002/(SICI)10990984(199811/12)12:6<411::AID-PER317>3.0.CO;2-C

DaSilveira, A., DeCastro, T. G., \& Gomes, W. B. (2012). Escala de Autorreflexão e Insight: Nova Medida de Autoconsciência Adaptada e Validada para Adultos Brasileiros. Psico, 43(2), 155-162. Recuperado de http:// revistaseletronicas.pucrs.br/ojs/index.php/revistapsico/article/viewFile/11693/8039

DaSilveira, A., DeSouza, M. L., \& Gomes, W. B. (2015). Self-consciousness concept and assessment in self-report measures. Frontiers in Psychology, 6(930), 1-11. https://doi.org/10.3389/fpsyg.2015.00930

DeSouza, M. L. \& Gomes, W. B. (2005). Aspectos históricos e contemporâneos na investigação do self. Memorandum, 9, 78-90. Recuperado de http://www.fafich.ufmg.br/ memorandum/a09/souzagomes01.pdf.

Eng, C.-J. \& Pai, H.-C. (2015). Determinants of nursing competence of nursing students in Taiwan: The role of self-reflection and insight. Nurse Education Today, 35(3), 450-455. https://doi.org/10.1016/j.nedt.2014.11.021

Fenigstein, A., Scheier, M. F., \& Buss, A. H. (1975). Public and Private Self-Consciousness: Assesment and Theory. Journal of Consulting and Clinical Psychology, 43(4), 522-527. https://doi.org/10.1037/h0076760

Gerace, A. \& Day, A. (2010). Criminal Rehabilitation: The Impact of Religious Programming. Journal of Psychology and Christianity, 29(4), 317-325.

Grant, A. M. (2001). Rethinking Psychological Mindedness: Metacognition, Self-reflection, and Insight. Behaviour Change, 8(18), 8-17. https://doi.org/10.1375/bech.18.1.8

Grant, A. M. (2003). The impact of life coaching on goal attainment, metacognition and mental health. Social Behavior and Personality, 31(3), 253-264. https://doi.org/10.2224/sbp.2003.31.3.253

Grant, A. M., Franklin, J., \& Langford, P. (2002). The self-reflection and insight scale: A new measure of private selfconsciousness. Social Behavior and Personality: an International Journal, 30(8), 821-835. https://doi.org/10.2224/ sbp.2002.30.8.821

Haga, S. M., Kraft, P., \& Corby, E-K. (2009). Emotion Regulation: Antecedents and Well-Being Outcomes of Cognitive Reappraisal and Expressive Suppression in Cross-Cultural Samples. Journal of Happiness Studies, 10(3), 271-291. https://doi.org/10.1007/s10902-007-9080-3

Harrington, R. \& Loffredo, D. A. (2011). Insight, Rumination, and Self-Reflection as Predictors of Well-Being. The Journal of Psychology, 145(1), 39-57. https://doi.org/10.1080/00223980.2010.528072

Harrington, R., Loffredo, D. A., \& Perz, C. A. (2016). Dispositional mindfulness facets and self-insight as predictors of subjective well-being: an exploratory analysis. North American Journal of Psychology, 18(3), 469-482.

Hays, R. B., Jolly, B. C., Caldon, L. J. M., McCrorie, P., McAvoy, P. A., McManus, I. C., \& Rethans, J. J. (2002). Is insight important? Measuring capacity to change performance. Medical Education, 36(10), 965-971. https://doi org/10.1046/j.1365-2923.2002.01317.x/full

Joireman, J. A., Parrot, L., \& Hammersla, J. (2002). Empathy and the Self-Absorption Paradox: Support for the Distinction Between Self-Rumination and Self-Reflection. Self and Identity, 1, 53-65. https://doi. org/10.1080/152988602317232803

Koriat, A. (2006). Metacognition and consciousness. In P. D. Zelazo, M. Moscovitch, \& E. Thompson (Eds.). Cambridge Handbook of Consciousness (pp. 289-325). New York, USA: Cambridge University Press.

Kounios, J. \& Beeman, M. (2009). The Aha! Moment. The Cognitive Neuroscience of Insight. Current Directions in Psychological Science, 18(4), 210-216. https://doi.org/10.1111/j.1467-8721.2009.01638.x

Lai, E. R. (2011). Metacognition: A Literature Review (Research Report). Pearson. Recuperado de http://images. pearsonassessments.com/images/tmrs/metacognition_literature_review_final.pdf.

Lyke, J. A. (2009). Insight but not self-reflection, is related to subjective well-being. Personality and Individual Differences, 46(1), 66-70. https://doi.org/10.1016/j.paid.2008.09.010 
Mass, M. J. M., van der Wees, P. J., Braam, C., Koetsenruijter, J., Heerkens, Y. F., van der Vleuten, C. P. M., \& Nijhuivan der Sanden, M. W. G. (2015). An innovative peer assessment approach to enhance guideline adherence in physical therapy: single-masked, cluster-randomized controlled trial. Physical Therapy, 95(4), 600-612. https://doi. org/10.2522/ptj.20130469

Pai, H.-C. (2015). The Effect of a Self-reflection and Insight program on the nursing competence of nursing students: a longitudinal study. Journal of Professional Nursing, 31(5), 424-431. https://doi.org/10.1016/j.profnurs.2015.03.003

Richards, K. C., Campenni, C. E., \& Muse-Burke, J. L. (2010). Self-care and Well-being in Mental Health Professionals: The Mediating Effects of Self-awareness and Mindfulness. Journal of Mental Health Counseling, 32(3), $247-264$. https://doi.org/10.17744/mehc.32.3.0n31v88304423806

Roberts, C. \& Stark, P. (2008). Readiness for self-directed change professional behaviours: factorial validation of the Self-reflection and Insight Scale. Medical Education, 42(11), 1054-1063. https://doi.org/10.1111/j.13652923.2008.03156.x

Sauter, F. M., Heyne, D., Blöte, A. W., van Widenfelt, B. M., \& Westenberg, P. M. (2010). Assessing TherapyRelevant Cognitive Capacities in Young People: Development and Psychometric Evaluation of the Self-Reflection and Insight Scale for Youth. Behavioural and Cognitive Psychotherapy, 38(3), 303-317. https://doi.org/10.1017/ S1352465810000020

Scheier, M. F. \& Carver, C. S. (1985). The Self-Consciousness Scale: A Revised Version for Use with General Populations. Journal of Applied Social Psychology, 15(8), 687-699. https://doi.org/10.1111/j.1559-1816.1985. tb02268.x

Schraw, G. \& Moshman, D. (1995). Metacognitive Theories. Educational Psychology Review, 7(4), 351-371. https:// doi.org/10.1007/BF02212307

Silvia, P. J. (1999). Explaining Personality or Explaining Variance? A Comment on Creed and Funder (1998). European Journal of Personality, 13(6), 533-538. https://doi.org/10.1002/(SICI)1099-0984(199911/12)13:6<533::AIDPER354>3.3.CO;2-S

Silvia, P. J. \& Duval, T. S. (2001). Objective self-awareness theory: Recent progress and enduring problems. Personality and Social Psychology Review, 5(3), 230-241. https://doi.org/10.1207/S15327957PSPR0503_4

Silvia, P. J. \& Phillips, A. G. (2011). Evaluating self-reflection and insight as self-conscious traits. Personality and Individual Differences, 50(2), 234-237. https://doi.org/10.1016/j.paid.2010.09.035

Snyder, M. (1974). Self-monitoring of expressive behavior. Journal of Personality and Social Psychology, 30(4), 526-537. https://doi.org/10.1037/h0037039

Stein, D. \& Grant, A. M. (2014). Disentangling the relationships among Self-Reflection, Insight, and Subjective WellBeing: The role of dysfunctional attitudes and core Self-Evaluations. The Journal of Psychology, 148(5), 505-522. https://doi.org/10.1080/00223980.2013.810128

Sutton, A. (2016). Measuring the Effects of Self-Awareness: Construction of the Self-Awareness Outcomes Questionnaire. Europe's Journal of Psychology, 12(4), 645-658. https://doi.org/10.5964/ejop.v12i4.1178

Trapnell, P. D. \& Campbell, J. D. (1999). Private Self-Consciousness and the Five-Factor Model of Personality: distinguishing Rumination From Reflection. Journal of Personality and Social Psychology, 76(2), 284-304. https:// doi.org/10.1037//0022-3514.76.2.284

van Dulmen, S. A., Mass, M. J. M., Staal, J. B., Rutten, G., Kiers, H., Nijhui-van der Sanden, M. W. G., \& van der Wees, P. J. (2014). Effectiveness of peer assessment for implementing a dutch physical therapy low back pain guideline: cluster randomized controlled trial. Physical Therapy, 94(10), 1396-1409. https://doi.org/10.2522/ptj.20130286

Watson, P. J., Morris, R. J., Ramsey, A., Hickman, S. E., \& Waddell, M. G. (1996). Further contrasts between selfreflectiveness and internal state awareness factors of private self-consciousness. The Journal of Psychology, 130(2), 183-192. https://doi.org/10.1080/00223980.1996.9915000

Xu, X. (2011). Self-Reflection, Insight, and Individual Differences in Various Language Tasks. The Psychological Record, 61(1), 41-58. Recuperado de http://opensiuc.lib.siu.edu/cgi/viewcontent.cgi?article=1377\&context=tpr. https://doi.org/10.1007/BF03395745

Zanon, C. \& Teixeira, M. A. P. (2006). Adaptação do Questionário de Ruminação e Reflexão (QRR) para estudantes universitários brasileiros. Interação em Psicologia, 10(1), 75-82. https://doi.org/10.5380/psi.v10i1.5771

Agradecimento:

O presente trabalho foi realizado com apoio da Coordenação de Aperfeiçoamento de Pessoal de

Nível Superior - Brasil (CAPES) - Código de Financiamento 001.

Dados dos autores:

Cristyan Karla Nogueira Leal - Mestra, Universidade Federal do Espírito Santo.

Maria Dolores Pinheiro de Souza - Doutoranda, Universidade Federal do Espírito Santo.

Mariane Lima de Souza - Doutora, Universidade Federal do Espírito Santo.

Endereço para correspondência:

Mariane Lima de Souza

Rua Genserico Encarnação, 185/304C - Mata da Praia

29065-420 Vitória, ES, Brasil

$<$ leal.ckn@gmail.com>

Recebido em: 25.02.2017

Aceito em: 29.05.2017 